\title{
Conformation of Tunable Nanocylinders: Up to Sixth-Generation Dendronized Polymers via Graft-Through Approach by ROMP
}

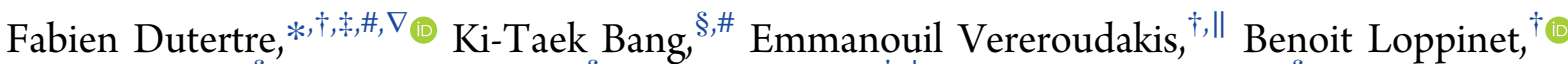 \\ Sanghee Yang, ${ }^{\S}$ Sung-Yun Kang, ${ }^{\S}$ George Fytas, ${ }^{*}, \dagger, \perp$ and Tae-Lim Choi* ${ }^{*}{ }^{\S}(0)$ \\ ${ }^{\dagger}$ Institute of Electronic Structure and Laser, FO.R.T.H, PO Box 1527, 71110 Heraklion, Greece \\ ${ }^{\ddagger}$ School of Chemistry, University of Bristol, Cantock’s Close, Bristol BS8 1TS, U.K. \\ ${ }^{\S}$ Department of Chemistry, Seoul National University, Seoul 08826, Republic of Korea \\ "Department of Materials Science \& Technology, University of Crete, 71003 Heraklion, Crete, Greece \\ ${ }^{\perp}$ Max Planck Institute for Polymer Research, Ackermannweg 10, 55128 Mainz, Germany
}

Supporting Information

ABSTRACT: Well-defined dendronized polymers (denpols) bearing high-generation dendron are attractive nano-objects as high persistency provides distinct properties, contrast to the random coiled linear polymers However, their syntheses via graft-through approach have been very challenging due to their structural complexity and steric hindrance retarding polymerization. Here, we report the first example of the synthesis of poly(norbornene) (PNB) containing ester dendrons up to the sixth generation (G6) by ring-opening metathesis polymerization. This is the highest generation ever polymerized among dendronized polymers prepared by graftthrough approach, producing denpols with molecular weight
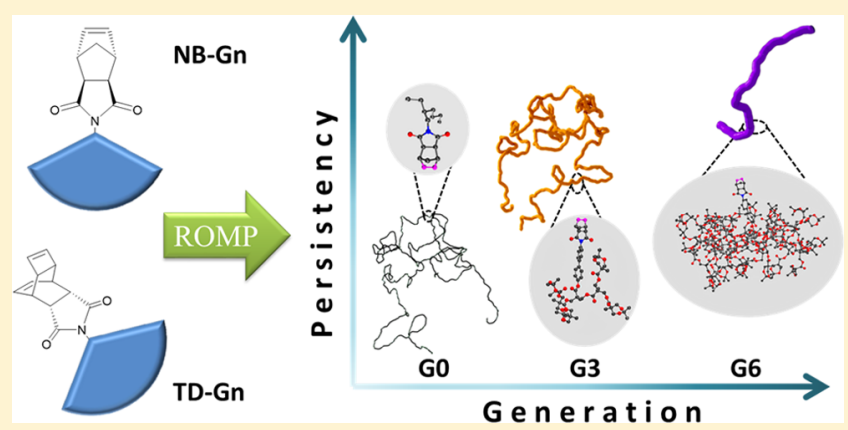
up to $1960 \mathrm{~kg} / \mathrm{mol}$. Combination of size-exclusion chromatography, light scattering, and neutron scattering allowed a thorough structural study of these large denpols in dilute solution. A semiflexible cylinder model was successfully applied to represent both the static and dynamic experimental quantities yielding persistent length $\left(l_{\mathrm{p}}\right)$, cross-sectional radius $\left(R_{\mathrm{cs}}\right)$, and contour length $(L)$. The denpol persistency seemed to increase with generation, with $l_{\mathrm{p}}$ reaching $27 \mathrm{~nm}$ (Kuhn length $54 \mathrm{~nm}$ ) for PNBG6, demonstrating a rod-like conformation. Poly(endo-tricycle[4.2.2.0]deca-3,9-diene) (PTD) denpols exhibited larger persistency than the PNB analogues of the same generation presumably due to the higher grafting density of the PTD denpols. As the dendritic side chains introduce shape anisotropy into the denpol backbone, future work will entail a study of these systems in the concentrated solutions and melts.

\section{INTRODUCTION}

The ability to systematically synthesize macromolecules with well-defined architecture has long been a goal of polymer chemistry as macromolecules with specific functionalities and complex architectures can possess unique properties. This is, for example, the case of graft or bottlebrush polymers, a class of branched macromolecules consisting of linear polymers as side chains grafted on the main-chain polymers. They exhibited unique properties with potential applications in the field of photonic crystals, ${ }^{1}$ photolithograpy, ${ }^{2}$ and biological elastomers. ${ }^{3}$ However, a precise control on the polymer architecture is still elusive due to polydispersity and defects issues of the polymeric side chains.

Dendronized polymers (denpols) are another class of branched macromolecules bearing unique dendritic side chains. It has attracted great interest stemmed from the expectation that they combine properties of grafted polymers and dendrimers, which have highly dense multifunctional groups at the periphery and intramolecular cavities leading to high solubility and low viscosities. ${ }^{4}$ One of the most interesting features of denpols, distinct from conventional linear polymers, is their extended or persistent conformation due to large steric repulsion among the side chains. ${ }^{5,6}$ Hence, denpols possess rich perspectives for applications including drug delivery, ${ }^{7}$ surface coating, ${ }^{8}$ photonic crystals, ${ }^{9}$ and molecular assemblies. ${ }^{10}$ However, to what extent the polymer chains extend is often overlooked in most applications, ${ }^{7-10}$ although chemists would agree that the denpols could transform from Gaussian coil to rod-like structures by increasing the generation of the dendron. ${ }^{11}$ The connection between dendron size (crosssectional radius) and chain rigidity has been rarely investigated. $^{12,13}$

It leads us to study the chain conformation in a systemic manner. Denpols are suitable for the investigation since their

Received: March 7, 2019

Revised: April 5, 2019

Published: April 23, 2019 
Scheme 1. Synthesis of the Denpols via ROMP Using Various Monomers and Catalysts

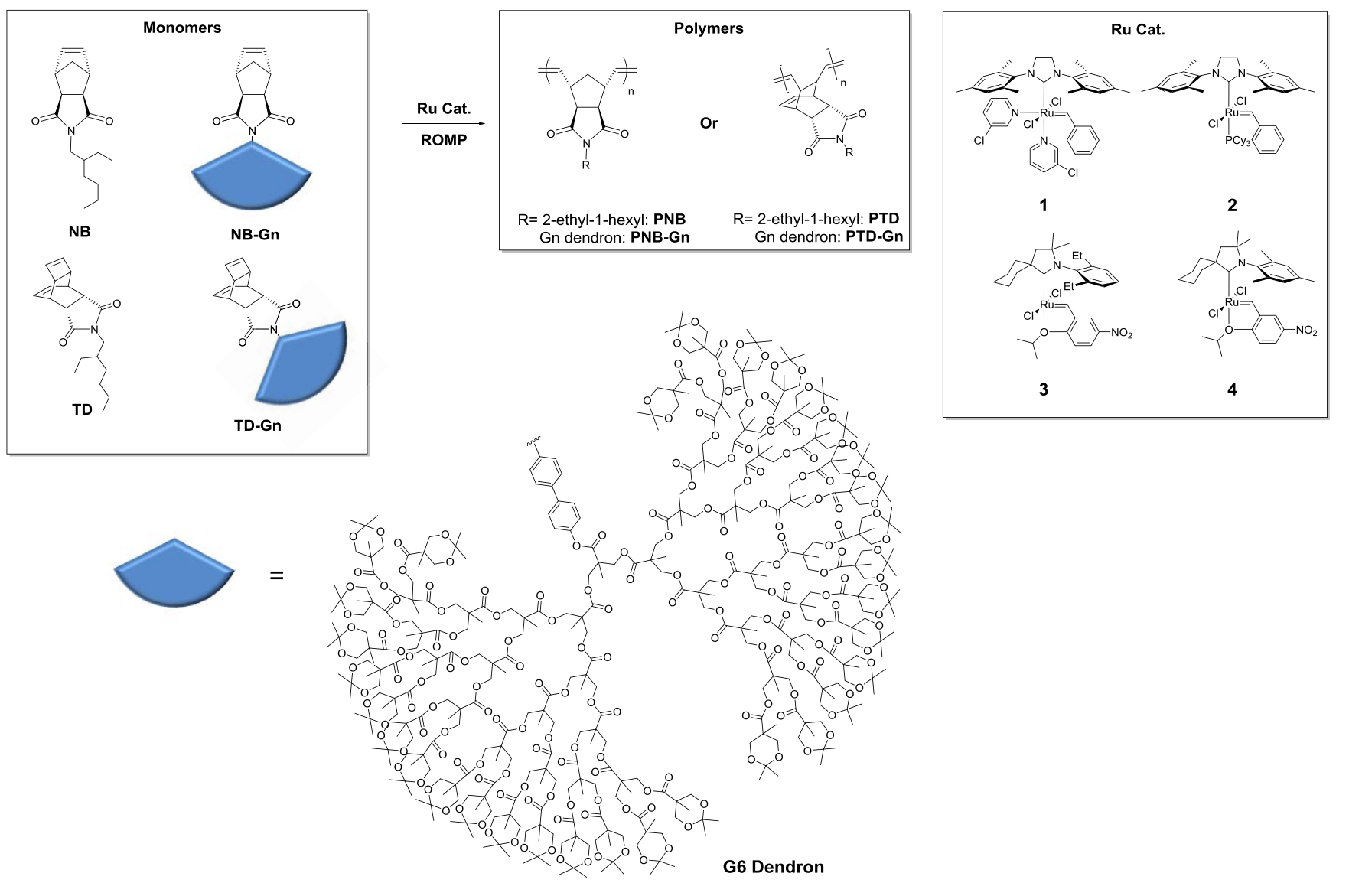

excellent control on the side chains at the molecular level can eliminate dispersity and defects issues, which are inevitable for graft polymers. Therefore, it is much easier to modulate their cross-sectional profile and conformation by tuning the sizes of the side-chain dendrons with high fidelity and precision. In this context, recent theoretical work suggests that the main difference between denpols and graft polymers ${ }^{14}$ should arise from the higher density in dendritic architectures compared to linear polymer chains.

Persistence length $\left(l_{\mathrm{p}}\right)$ has been mostly used for quantifying the conformational rigidity of the polymer backbone and can be controlled by grafting density, size of the graft, and length of the main-chain backbone. ${ }^{15}$ Although atomic force microscopy (AFM) analysis could determine $l_{\mathrm{p}}$ of denpols, ${ }^{16}$ one should be aware that AFM can overestimate because adsorption could induce stretching on the substrates. ${ }^{17}$ Alternatively, scattering techniques $^{12}$ have been used for more reliable estimation of $l_{\mathrm{p}}$ in solution, eliminating surface effects. For instance, $l_{\mathrm{p}}$ ranges between 1 and $10 \mathrm{~nm}$ in polymers containing up to secondgeneration $^{18}$ carbosilane dendrons. On the other hand, $l_{\mathrm{p}}$ of 30 $\mathrm{nm}$ was reported for the denpol containing fifth-generation aromatic amide dendrons. ${ }^{12,13}$ We have reported $l_{\mathrm{p}}$ values around $6-8 \mathrm{~nm}$ for polynorbornene (PNB) and poly(endotricycle[4.2.2.0] deca-3,9-diene) (PTD) containing third-generation ester dendrons in dilute regime by the combination of static light scattering (SLS) and dynamic light scattering (DLS). ${ }^{19}$ However, the impact of even higher dendron generation on the denpols conformation still remains largely unexplored for denpols, obtained by graft-through approach, due to the lack of good synthetic access to them.
The main obstacle in the synthesis of large denpols is the precise installation of the bulky side chains with high fidelity. To tackle this problem, many approaches have been investigated for the preparation of well-defined denpols. For example, grafting-to method ${ }^{20-25}$ (coupling dendrons to the precursor polymers directly) or most widely used grafting-from approach $^{26-30}$ (step by step dendronization of every generation onto the precursor polymer chain) has been successful to prepare denpols carrying up to eighth-generation dendrons. ${ }^{30,31}$ However, these methods lead to some defects, and analysis to confirm their structure can be tedious, especially for high generations. The alternative approach is grafting-through or macromonomer method ${ }^{32-42}$ via directly polymerizing well-defined macromonomers containing the defect-free dendrons. Although this allows the synthesis of highly pure denpols, it is the most challenging method as the steric hindrance between the bulky dendrons greatly retards the polymerization. Fortunately, recent advances of ringopening metathesis polymerization (ROMP) $)^{43-45}$ have provided an excellent solution to overcome this challenge. For example, we reported denpols of poly(endotricycle[4.2.2.0]deca-3,9-diene) (PTD) containing up to fourth-generation dendrons ${ }^{42}$ and poly(norbornene) (PNB) containing up to fifth-generation dendrons, ${ }^{35}$ as well as their block copolymers and gradient copolymers ${ }^{42}$ by ROMP. Therefore, we envisioned that the synthetic efforts toward even larger denpols would certainly get a positive feedback from a thorough study of their conformation in solution.

Herein, we report the synthesis of large denpols (up to G6 on PNB and G5 on PTD, Scheme 1) having molecular weight 
Table 1. Characterizations of the Polymers

\begin{tabular}{|c|c|c|c|c|c|c|c|}
\hline \multicolumn{4}{|c|}{ PNB } & \multicolumn{4}{|c|}{ PTD } \\
\hline entry & name $^{a}$ & $M_{\mathrm{w}}{ }^{b}(\mathrm{~kg} / \mathrm{mol})$ & $\boxplus^{b}$ & Entry & name $^{a}$ & $M_{\mathrm{w}}^{b}(\mathrm{~kg} / \mathrm{mol})$ & $\boxplus^{b}$ \\
\hline 1 & PNB-287 & 79 & 1.06 & 14 & PTD-931 & 292 & 1.16 \\
\hline 2 & PNB-1587 & 437 & 1.19 & & & & \\
\hline 3 & PNB-G3-510 & 666 & 1.26 & 15 & PTD-G3-1060 & 1424 & 1.25 \\
\hline 4 & PNB-G3-750 & 978 & 1.55 & 16 & PTD-G3-1376 & 1847 & 1.20 \\
\hline 5 & PNB-G3-1257 & 1640 & 1.48 & & & & \\
\hline 6 & PNB-G4-301 & 720 & 1.25 & 17 & PTD-G4-257 & 626 & 1.17 \\
\hline 7 & PNB-G4-475 & 1136 & 1.37 & 18 & PTD-G4-630 & 1532 & 1.15 \\
\hline 8 & PNB-G5-148 & 678 & 1.28 & $19^{c}$ & PTD-G5-205 & 938 & 1.39 \\
\hline 9 & PNB-G5-208 & 949 & 1.45 & $20^{d, e}$ & PTD-G5-369 & 1699 & 1.41 \\
\hline 10 & PNB-G5-295 & 1350 & 1.76 & & & & \\
\hline $11^{c}$ & PNB-G6-78 & 698 & 1.45 & & & & \\
\hline $12^{c}$ & PNB-G6-128 & 1145 & 1.77 & & & & \\
\hline $13^{d}$ & PNB-G6-220 & 1960 & 1.33 & & & & \\
\hline
\end{tabular}

${ }^{a}$ Numbers at the end of the label indicate calculated $N_{\mathrm{w}}{ }^{b} M_{\mathrm{w}}$ and $Ð$ were determined by SEC-MALLS in chloroform. ${ }^{c}$ Third-generation Grubbs catalyst (2) was used. ${ }^{d}$ The polymer was fractionated using preparative-SEC. ${ }^{e}$ Catalyst 4 was used.

up to $1960 \mathrm{kDa}$ along with their unique conformational analysis in solution. The consistent determination of the configurational quantities, obtained from the combination of size-exclusion chromatography multiangle laser light scattering (SEC-MALLS), static and dynamic light scattering (SLS/ DLS), and small-angle neutron scattering (SANS) techniques, revealed the effect of backbone structure and generation on the conformation. The high persistence $l_{\mathrm{p}}$ up to $27 \mathrm{~nm}$ strongly suggests a rod-like conformation of the high-generation denpols.

\section{RESULTS}

II.I. Synthesis. To achieve well-defined denpols by graftthrough approach, we selected highly reactive monomers such as $\mathrm{NB}^{35}$ and TD. ${ }^{42}$ The release of their ring strain is essential to overcome the steric hindrance during propagation of a polymer chain. We also introduced the rigid biphenyl spacer between the active monomer and dendron, which is necessary to reduce the steric hindrance during the propagation, compromising slight loss of chain rigidity. ${ }^{35,42}$ Recently, we have reported on the conformation of denpols containing G0 and G3 dendron using static and dynamic light scattering ${ }^{19}$ (Table 1, entries $1-5$ and 15-16). Since the steric repulsion from the medium-size G3 dendron was moderate, these denpols exhibited semiflexible chain structure. To examine the conformation of larger denpols of higher generation, we prepared PNB with G4 and G5 ester dendrons and PTD with G4 dendron using Grubbs third-generation catalyst $(\mathbf{1})^{35}$ (Table 1, entries 6-10 and 17-18). PTD containing 2-ethyl-1hexyl side chain ${ }^{46}$ was prepared as a reference material for PTD-Gn (entry 14, Table 1). We envisioned that comparison of the PNB and PTD chain conformation would lead to a deeper insight into the effect of the backbone structures. The obtained polymers have weight-average molecular weights $\left(M_{\mathrm{w}}\right)$ ranging from 292 to $1350 \mathrm{~kg} / \mathrm{mol}$ and relatively narrow dispersity except entry 10 .

To push the limit of the synthesis beyond the state of the art, we attempted to synthesize PTD containing G5 dendron and even larger PNB containing the largest G6 dendron via ROMP. Synthesis of these extremely large denpols was extremely challenging as such huge denpols have not been prepared by graft-through approach. The synthesis of the monomers NBG6 and TD-G5 was successfully achieved by following the reported procedure, ${ }^{35}$ and their structures were confirmed by NMR and matrix-assisted laser desorption/ionization mass spectroscopy (see Figures S3-S8). However, as anticipated, initial attempts to polymerize TD-G5 and NB-G6 by using 1 did not yield the desired polymers even at $50{ }^{\circ} \mathrm{C}$ presumably due to the low reactivity of the congested propagating species. On the other hand, ROMP of NB-G6 using highly active and thermally stable second-generation Grubbs catalyst (2) in toluene at $70{ }^{\circ} \mathrm{C}$ afforded the denpols with moderate weight average degree of polymerization $\left(N_{\mathrm{w}}=M_{\mathrm{w}} / M_{0}\right.$, where $M_{0}$ is the molecular weight of a monomer) of 78 and dispersity of 1.45 (Table 1, entry 11).

Encouraged by this initial result, we further optimized this ROMP in THF at $60{ }^{\circ} \mathrm{C}$, where the faster ROMP was reported compared to toluene ${ }^{47}$ and the $M_{\mathrm{w}}$ increased to $1145 \mathrm{~kg} / \mathrm{mol}$ with $N_{\mathrm{w}}$ of 128 (Table 1, entry 12). Further trials to obtain higher-molecular-weight polymers by increasing reaction concentration, changing the solvent from THF to 1,2dichloroethane (DCE) and increasing temperature to $70{ }^{\circ} \mathrm{C}$, were not satisfactory (see Table S1).

As an alternative strategy, we switched the catalyst to the recently developed $\mathrm{Ru}$ catalysts containing cyclic (alkyl)(amino)carbine (CAAC), which showed even higher activity toward ring-closing metathesis (RCM $)^{48}$ and cross-metathesis $(\mathrm{CM})^{49}$ reaction, hoping that it would do the same to ROMP. Indeed, after several trials using several catalysts including 3, we obtained the denpols having larger fraction of high molecular weight despite the broader dispersity (see Table S1), and fortunately, fractionating out their low-molecularweight portion via preparative-SEC successfully provided the denpol having $M_{\mathrm{w}}$ of $1960 \mathrm{~kg} / \mathrm{mol}$ with $N_{\mathrm{w}}$ of 220 and narrow dispersity (Table 1, entry 13) (see Table S1). Notably, this enables us to explore the behavior of high-molecular-weight G6 denpols in the dilute solution for the first time. Likewise, using the new optimized condition to prepare PNB-G6, ROMP of TD-G5 using G2 in THF at $60{ }^{\circ} \mathrm{C}$ afforded the PTD-G5 with high $M_{\mathrm{w}}$ of $938 \mathrm{~kg} / \mathrm{mol}, N_{\mathrm{w}}$ of 205 , and moderate dispersity of 1.39 (Table 1 , entry 19). Furthermore, using CAAC catalyst (4) and fractionalization (see Table S2) afforded the PTD-G5 denpols having even higher $M_{\mathrm{w}}$ of 1699 $\mathrm{kg} / \mathrm{mol}$ corresponding to $N_{\mathrm{w}}$ of 369 with moderate dispersity (Table 1, entry 20). 

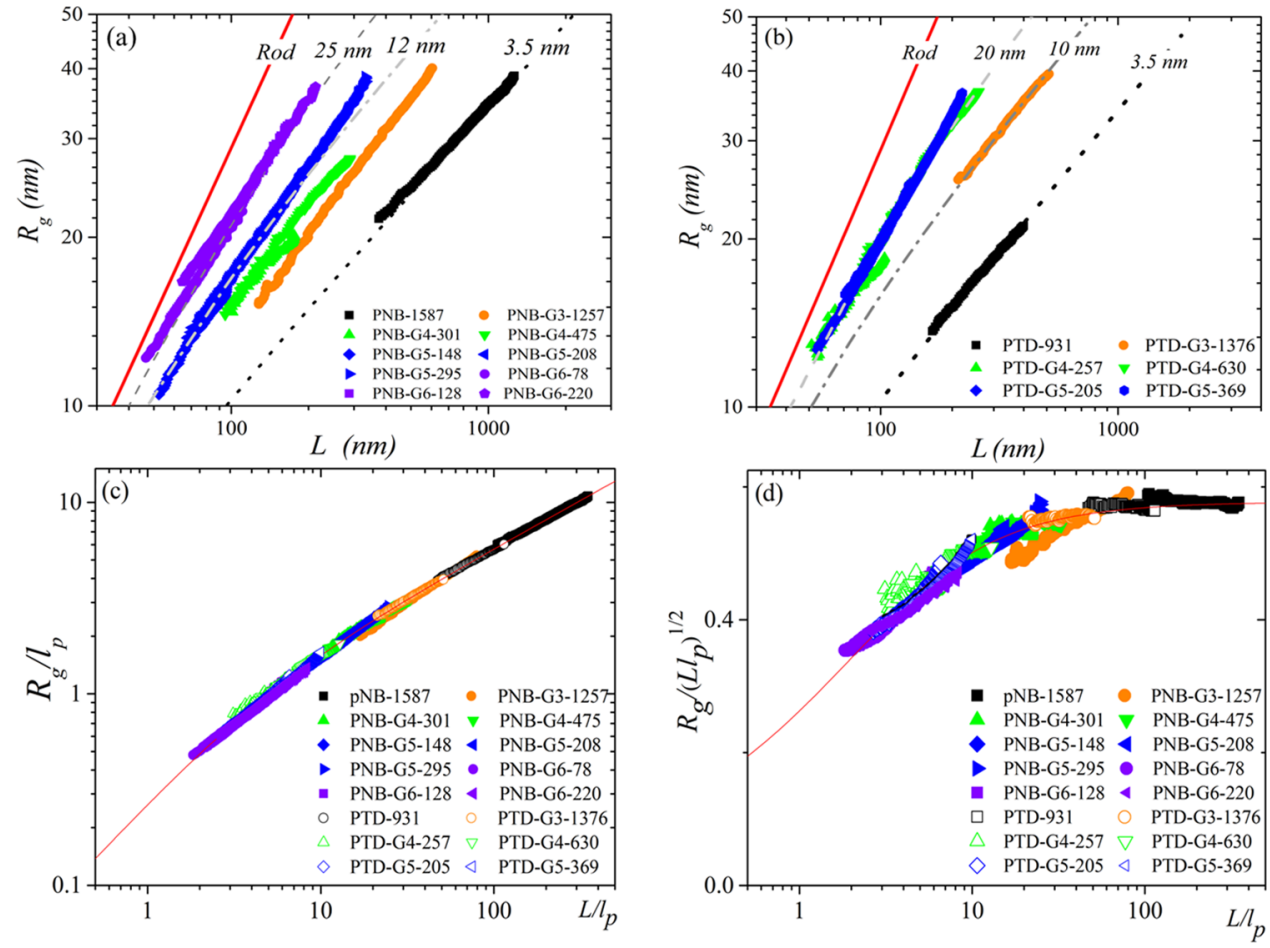

Figure 1. Radius of gyration $\left(R_{\mathrm{g}}\right)$ as a function of weight-averaged contour length $(L)$, obtained from MALLS-SEC measurement, in THF, for PNB-Gn denpols (a) and PTD-Gn denpols (b). The various dashed lines represent the Benoit-Doty model (eq S1) for different values of persistence length. The red solid lines in (a) and (b) represent the rod scaling behavior. The dependence of the radius of gyration contour length in Benoit-Doty master presentation: Double-logarithmic plot (c) and semilog presentation (d). The plateau region in (d) indicates the flexible coil limit, $R_{\mathrm{g}} \sim\left(L / l_{\mathrm{p}}\right)^{1 / 2}$.

Table 2. Molecular Parameters (Contour Length, $L$; Radius of Gyration, $R_{\mathrm{g}}$; Hydrodynamic Radius, $R_{\mathrm{h}}$; and Persistence Length, $l_{\mathrm{p}}$ ) of Selected Polymers in Dilute Solutions in THF

\begin{tabular}{|c|c|c|c|c|c|c|c|c|}
\hline \multirow[b]{2}{*}{ code } & \multirow{2}{*}{$\frac{\text { DLS }}{R_{\mathrm{h}}(\mathrm{nm})}$} & \multicolumn{3}{|c|}{ SANS } & \multicolumn{4}{|c|}{ GPC-MALLS } \\
\hline & & $L_{\mathrm{K}}{ }^{a}(\mathrm{~nm})$ & $l_{\mathrm{K}}^{a}(\mathrm{~nm})$ & $R_{\mathrm{cs}}{ }^{a}(\mathrm{~nm})$ & $N_{\mathrm{w}}$ & $L(\mathrm{~nm})$ & $l_{\mathrm{p}}^{b}(\mathrm{~nm})$ & $R_{\mathrm{g}}(\mathrm{nm})$ \\
\hline PNB-287 & 8.6 & $93 \pm 5$ & $2.0 \pm 0.5$ & N/A & 370 & $185 \pm 9$ & $c$ & $c$ \\
\hline PNB-G3-1257 & 32.6 & $698 \pm 35$ & $6.0 \pm 0.8$ & 1.8 & 1182 & $591 \pm 30$ & 9.9 & 52.1 \\
\hline PNB-G4-301 & 19.6 & $240 \pm 12$ & $6.4 \pm 0.8$ & 1.9 & 313 & $157 \pm 8$ & 8.0 & 19.5 \\
\hline PNB-G5-295 & 24.2 & $217 \pm 11$ & $10.2 \pm 1.0$ & 2.3 & 271 & $135 \pm 7$ & 13.5 & 26.8 \\
\hline PNB-G6-128 & 15.4 & $75 \pm 4$ & $15.0 \pm 1.5$ & 2.8 & 134 & $67 \pm 3$ & 27.3 & 24.9 \\
\hline PTD-G3-1060 & 17.8 & $169 \pm 9$ & $13.7 \pm 1.4$ & 2.2 & 1444 & $534 \pm 27$ & 11.3 & 47.7 \\
\hline PTD-G4-630 & 16.0 & $113 \pm 6$ & $24.2 \pm 2.4$ & 2.4 & 600 & $222 \pm 11$ & 20.1 & 35.6 \\
\hline PTD-G5-205 & 16.3 & $65 \pm 3$ & $14.3 \pm 1.4$ & 2.8 & 175 & $65 \pm 3$ & 20.2 & 18.1 \\
\hline
\end{tabular}

${ }^{a}$ Contour length, $L_{\mathrm{K}}$; cross-sectional radius, $R_{\mathrm{cs}}$; and persistence length, $l_{\mathrm{K}}$ were obtained from the representation of the SANS form factor by Kholodenko model. ${ }^{50,51} b^{b}$ Obtained from SEC-MALLS data fitting using the Benoit-Doty equation (eq 1 in the SI). ${ }^{c} R_{\mathrm{g}}(<10 \mathrm{~nm})$ is too small to be measured by MALLS detector.

II.II. Persistence Length from SEC MALLS $R_{\mathrm{g}}$ vs M. To systematically investigate the relationship between dendron generation and conformation, we analyzed the denpols by MALLS, providing the $M_{\mathrm{w}}$ and the radius of gyration $\left(R_{\mathrm{g}}\right)$. The relationship between $M_{\mathrm{w}}$ and $R_{\mathrm{g}}$ provides a basis to extract persistence length $\left(l_{\mathrm{p}}\right)$ using standard wormlike chain models. These quantities are obtained on monodisperse elution by SEC-MALLS so that polydispersity effects can be ignored. We present $R_{\mathrm{g}}$ as a function of effective contour length $\left(L=b_{0} N_{w}\right)$, where $b_{0}$ is the length of a repeat unit. The evolution of $R_{\mathrm{g}}$ with $L$ within each fraction of the PNB and PTD denpols is depicted in the double-logarithmic plot (Figure 1a,b). With increasing generation in both denpols, the observed $R_{\mathrm{g}}(L)$ dependence becomes stronger (steeper slope), implying increase of $l_{\mathrm{p}}$.
The experimental data of Figure 1 were well represented (dashed lines) by the Benoit-Doty expression (see Section III.5.1. in the Supporting Information (SI)) using $l_{\mathrm{p}}$ as a single adjustable parameter. For all fractions, the experimental $R_{\mathrm{g}}$ values were well superimposed into two master curves representing both limiting rod and coiled behaviors in Figure 1c,d. For the rod-like regime, the scaling $R_{\mathrm{g}} \sim L^{1}$ was tentatively observed in the low $L / l_{\mathrm{p}}$ limit, while a flexible coil, $R_{\mathrm{g}} \sim L^{1 / 2}$, was obeyed at high $L / l_{\mathrm{p}}(>50)$. The distinct crossover from the rod-like to the flexible coil chain conformation according to the Benoit-Doty master plots (Figure 1d) occurred at higher $L$ (or $N_{\mathrm{w}}$ ) with increasing dendron generation, as revealed in Figure 1a,b. PNB-G6 and PTD-G5 approached the rod regime (red solid lines), whereas denpols with low generation falls near the flexible-chain regime 
(dotted black lines in Figure 1a,b). This general trend is consistently reflected in the increase of $l_{\mathrm{p}}$ (from 3.5 to $27 \mathrm{~nm}$ ) in THF, as listed in Table 2. The same analysis in chloroform solution gave the similar results (see Table S5), demonstrating their rod-like conformation. Moreover, based on the low but positive values of the second virial coefficient $\left(A_{2}<10^{-4} \mathrm{~mol}\right.$. $\mathrm{cm}^{3} \cdot \mathrm{g}^{-2}$ ), determined by SLS, both CF and THF display similar weak repulsive denpol-denpol interactions, implying similar solvent quality. AFM images showing single chains further support their rigid conformations inferred from the scattering data in solution (see Figures $2 \mathrm{c}$ and S18).
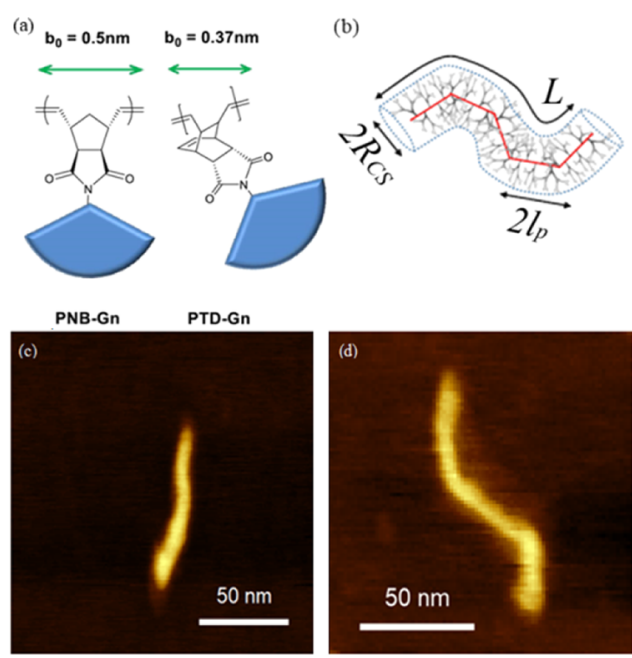

Figure 2. (a) Schematic representation of macromononers dimensions, (b) denpol chain within a wormlike conformation. (c) Representative AFM images of PNBG6-220 and (d) PTDG5-369.

II.III. SANS Patterns and Internal Structure. The emerged structure of the two denpols is schematically shown in Figure 2 . The cross-sectional radius, $R_{\mathrm{cs}}$, cannot be obtained by SEC-MALLS enabling access only to the low wave-vector fraction of the form factor sufficient to determine $R_{\mathrm{g}}$. To obtain the full form factor with access to the local structure of the denpols in dilute solutions, the higher resolution $\left(2 \times 10^{-3}\right.$ $\mathrm{nm}^{-1}$ ) offered by the SANS technique is necessary. The normalized intensity patterns for the PNB denpols in dilute THF solutions, from G0 to G6, are shown as $\log (I)$ vs $\log (q)$ in Figure $3 \mathrm{a}$ and $I \times q / I_{0}$ vs $\log q$ in Figure $3 \mathrm{~b}$. Analogous scattering curves recorded for various PTD are available in Figure S15. The scattering profile of PNB-G0 (black squares) conforms to the form factor of a flexible polymer chain with typical scaling $I(q) \sim q^{-2}$ at high $q$ 's, as indicated in Figure 3a. However, for the denpols, the form factor is distinctly different at high $q$ 's $\left(>0.6 \mathrm{~nm}^{-1}\right)$, exhibiting steeper decay, which is a sign of the finite cross-sectional dimension of denpols (Figure 3a). At intermediate wave-vectors, the characteristic $q^{-1}$ dependence of semirigid chains for $q l_{\mathrm{p}} \sim 1$ is observed, whereas for $q R_{\mathrm{g}}<1$, a low $q$ Guinier plateau is reached.

We used the Kholodenko form factor as it is recognized to be best adapted for the description of semiflexible cylinder (Figure 3). ${ }^{50-52}$ The representation requires an intensity $\left(I_{0}\right)$ prefactor and three size parameters, contour length $L_{\mathrm{K}}$, persistence length $l_{\mathrm{p}, \mathrm{K}}$ and cross-sectional radius $R_{\mathrm{cs}}$ (Figure 2b), as described in SI-II.5 (eqs 3-5). The experimental intensity patterns were well represented by the theoretical form factor (red dashed lines in Figure 3a). The quality of the Kholodenko model representation is better illustrated in the semilogarithmic plot $I . q / I_{0}$ vs $q$ (Figure $3 \mathrm{~b}$ ), and the values of the adjustable three size parameters are reported in Table 2. Notably, $L_{\mathrm{K}}$ and $l_{\mathrm{p}, \mathrm{K}}$ are in good agreement with the values of the corresponding parameters obtained from SEC-MALLS.

II.IV. Hydrodynamic Radius. Angular-dependent DLS measurements lead to the straightforward determination of diffusion coefficient $D$ and hydrodynamic radius $R_{\mathrm{h}}$. The experimental values of $R_{\mathrm{h}}$ for the different samples are reported in Table 2 . The hydrodynamic radius $R_{\mathrm{h}} \sim D^{-1}$ is expected to reflect the conformation and the hydrodynamic conditions of the denpols in dilute solutions. It is primarily sensitive to many factors such as the overall size of the denpol, rigidity of a polymer chain, thickness (cross-sectional radius, $R_{\mathrm{cs}}$ ), solvent quality, as well as the type of branching. ${ }^{53,54}$ However, the contribution of the different parameters is not straightforward to distinguish. ${ }^{55,56}$ Different models have been reported for the diffusion of semiflexible chains. We have chosen a model based on an accurate description of the polymer chain motion and standard hydrodynamic assumption (Zimm model) developed by Winkler et al. ${ }^{5,57}$ As in the case of the SANS, the representation of $D$ also requires the same three size parameters (contour length, persistence length, and hydrodynamic cross-sectional radius).
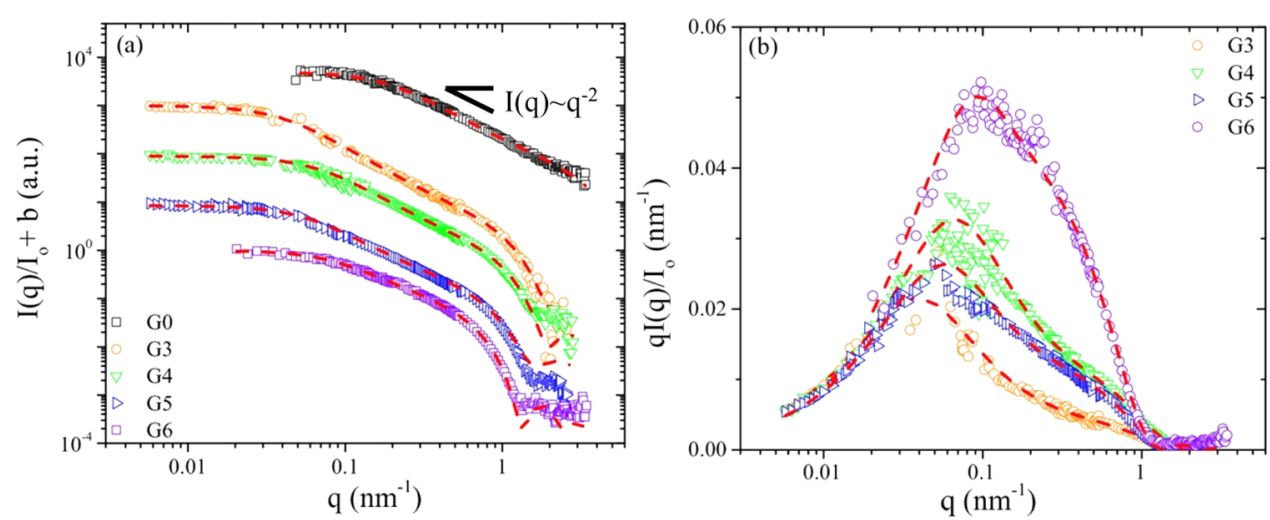

Figure 3. (a) Scattering intensities from the combined SANS and SLS experiments on PNB-Gn denpols in dilute THF- $d_{8}$ solution $(c=5 \mathrm{~g} / \mathrm{l})$ as a function of wave-vector $q$. The data along with their representation (dashed lines) have been shifted vertically for clarity. (b) Representation (red dashed lines) of the scattering intensity, normalized by the forward scattering intensity, and the wave-vector (for rods) by the Kholodenko form factor. $^{50-52}$ 
We utilized the values of $R_{\mathrm{cs}}$ and $l_{\mathrm{p}}$ obtained from MALLS and SANS to compute $R_{\mathrm{h}}$ as a function of $L$ using the Winkler model. ${ }^{57}$ Figure 4 compares the theoretical (green and purple

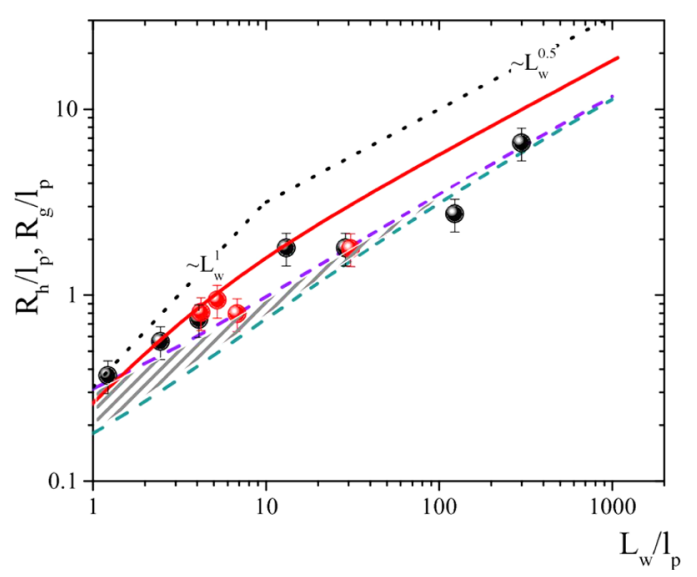

Figure 4. Hydrodynamic radius as a function of weight-averaged contour length for the two different backbones, PNB (black spheres) and PTD (red spheres), obtained from SLS/DLS experiments in THF and rescaled by $l_{\mathrm{p}}$ from SANS. The red line presents the radius of gyration variation from the Benoit-Doty equation. The dashed lines denote the theoretical prediction for the hydrodynamic radius for the upper $\left(l_{\mathrm{p}}=27.3 \mathrm{~nm}, R_{\mathrm{cs}}=2.8 \mathrm{~nm}\right.$, purple $)$ and lower limits of persistence length and cross-sectional radius $\left(l_{\mathrm{p}}=6.8 \mathrm{~nm}, R_{\mathrm{cs}}=1.8\right.$ $\mathrm{nm}$, green). Theoretical curves for thicknesses and rigidities as the ones measured experimentally fall in the marked area between the two curves. The black dotted lines show the contour length dependence for Gaussian coil $\left(R \sim L_{\mathrm{w}}^{0.5}\right)$ and rigid $\operatorname{rod}\left(R \sim L_{\mathrm{w}}^{1}\right)$.

dashed lines) with the measured $R_{\mathrm{h}}$ data as a function of the chain contour length. Both axes $R_{\mathrm{h}}$ and $L$ have been rescaled by the persistence length, to adopt a semiflexible chain master plot representation in analogy to Figure $1 \mathrm{c}, \mathrm{d}$ for $R_{\mathrm{g}}$. The evolution of the latter with length, also included (red line, Section II.5 in the SI, eq 2), is systematically smaller than $R_{\mathrm{h}}$. As expected at high $L / l_{\mathrm{p}}$ in the Gaussian coil limit (dashed lines in Figure 4$), R_{\mathrm{h}}$ follows a similar scaling to $R_{\mathrm{g}}\left(R_{\mathrm{g}} / R_{\mathrm{h}}=\right.$ 1.72 ), i.e., $R_{\mathrm{h}}(L)$ is equivalent to $R_{\mathrm{g}}(L)$. The agreement is moderate at low $L / l_{\mathrm{p}}$, possibly because the model is not well adapted for polymers with low $L / R_{\mathrm{cs}}$, i.e., for thick and short species. In this region, the simple $L / l_{\mathrm{p}}$ scaling does not hold any more and $R_{\mathrm{g}} / R_{\mathrm{h}}$ depends on the system specifics and the precise value of $R_{\mathrm{cs}}$ becomes crucial. Nevertheless, the crossover from rod-like scaling $R_{\mathrm{h}} \sim L_{\mathrm{W}}{ }^{1}$ and to flexible coil $R_{\mathrm{h}} \sim L_{\mathrm{W}}{ }^{1 / 2}$ scaling occurs around $L_{\mathrm{W}} / l_{\mathrm{p}} \sim 10$, consistent with the $R_{\mathrm{g}}$ data (Figure 1d). Hence, the persistence length can be also estimated from translational diffusion data, as it was reported in our previous work. ${ }^{19}$

\section{DISCUSSION}

Based on the simulation work on bottlebrush polymers, ${ }^{58,59} l_{\mathrm{p}}$ increases with the degree of polymerization of the side chains $\left(N_{s}\right)$ and counterintuitively with the main chain $(N)$. The dependence $l_{\mathrm{p}} \sim N_{\mathrm{s}}$ leads to deviation from the Kratky-Porod wormlike chain model. However, both predictions are not supported by the experimental evidence that $l_{\mathrm{p}}$ is robust over $N_{\mathrm{s}}$ and $N$ variations. For the present denpols, we utilized the wormlike chain model (WLC) consistently to represent form factor and translational diffusion. The former, obtained over broad wave-vector range, combining light and neutron scattering, was found to conform to the model including the denpols cross-sectional radius. The good agreement between GPC-MALLS, SANS and diffusion values supports the applicability of the WLC model in the case of the denpols. As a mesoscopic model cannot provide insight into the origin of the persistency, it cannot resolve the different possible contributions to denpols rigidity. The radial density profile cannot be resolved by SANS, but the appearance of a clear minimum at high $q$ 's (for the large generation) hints the formation of a steep concentration gradient. The consistent analysis of the results neither questions the validity of the WLC model to describe the conformation of the large Gn denpols in solutions nor provides insight into the origin of the measured "apparent" persistence length.

The study on the PNB and PTD systems yields insight into the effect of increasing dendron generation on the conformation of the denpols. Two factors can influence chain conformation: dendron molecular weight $\left(M_{\mathrm{sc}}\right)$ and branching. The former impacts the conformation since both $R_{\mathrm{cs}}$ and $l_{\mathrm{p}}$ increase with generation, as depicted in the log-log presentation of $R_{\mathrm{cs}}$ vs $M_{\mathrm{sc}}$ in Figure 5a. In the case of graft polymers in theta solvent, ${ }^{60} R_{\mathrm{cs}}$ is expected to grow as $M_{\mathrm{sc}}^{0.5}$. As to the effect of branching, Borisov et al. ${ }^{14}$ reported the scaling, $R_{\mathrm{cs}} \sim N_{\mathrm{sc}}^{0.75}$, with $N_{\mathrm{sc}}$ being the number of monomers in the side chain, in the presence of excluded volume interaction (good solvent), while $R_{\mathrm{cs}}$ is rather independent of the branching of
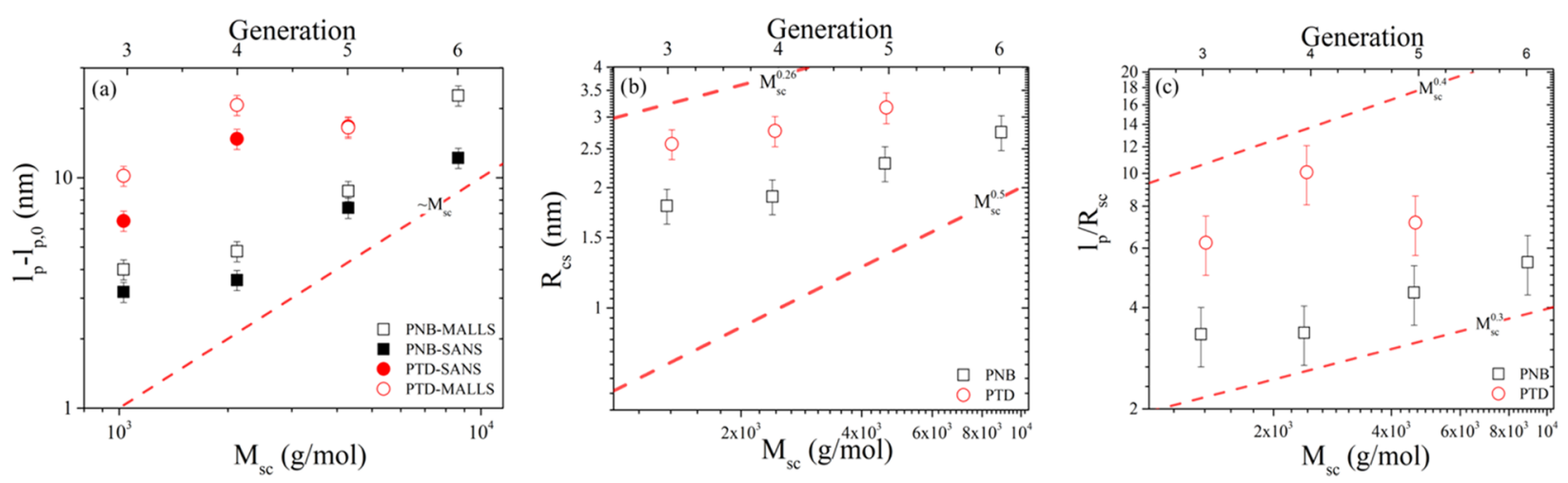

Figure 5. (a) Excess persistence length obtained from SANS (filled symbols) and MALLS-GPC analysis (opened symbols). The dashed line represents a linear scaling. $l_{\mathrm{p}, 0}$ is the persistence length of the linear chain. (b) A log-log plot of the cross-sectional radius vs dendron generation or equivalently the side-chain molecular weight. The two dashed lines represent different power law dependencies indicated in the plot. (c) Ratio of the persistence length to the cross-sectional radius as a function of generation in log-log presentation. 
the side chain. We note that there is a paucity of experimental results for validation of the theoretical prediction. In the present case, both PTD and PNB denpols show a rather weak increase of $R_{\mathrm{cs}}$ with generation, which conforms to $R_{\mathrm{cs}} \sim M_{\mathrm{sc}}^{0.25}$ and not to the brushlike $R_{\mathrm{cs}} \sim M_{\mathrm{sc}}^{0.5}$ dependence. The weak increase with generation indicates that the high-generation denpols are denser than the lower-generation denpols rendering their shape close to colloidal nano-objects. It might also imply dense packing near to the polymer main chain.

The persistence lengths obtained from SEC-MALLS (opened symbols) and from SANS (filled symbols) with each generation are reported in Figure $5 \mathrm{~b}$ as a function of $M_{\mathrm{sc}}$. The excess persistency $\left(l_{\mathrm{p}, 0}=3.5 \mathrm{~nm}\right.$ being the persistence length of the linear chain "precursor") increases with generation reaching the value of $27 \mathrm{~nm}$ for PNB-G6. The observed increase in $l_{\mathrm{p}}$ is almost linear to $M_{\mathrm{sc}}$. In fact, $l_{\mathrm{p}} \sim M_{\mathrm{sc}}$ was proposed by SCF calculation for bottlebrush polymers. ${ }^{6 \mathrm{C}}$

According to Borisov et al., ${ }^{14}$ this relationship should be robust over the structure of the side chain, implying that a linear side chain would increase the persistence length in the same way as a branched side chain with the same side-chain molecular weight. This conjecture concerning structural similarities between denpols and bottlebrush polymers remains to be examined.

Both $l_{\mathrm{p}}$ and $R_{\mathrm{cs}}$ are different for PNB and PTD bearing the same side chains (Figure 5a). The augmented disparity in the value of $l_{\mathrm{p}}$ is already evident in the form factors of PNB and PTD of G4 in the intermediate q-range (Figure S17). The scattering intensity from PTD-G4 with $l_{\mathrm{p}}=24 \mathrm{~nm}$ is higher than that for PNB-G4 with $l_{\mathrm{p}}=6.4 \mathrm{~nm}$. The PTD backbone is more persistent than the PNB backbone of the same generation, and the difference is more marked for high generations. ${ }^{62}$ The backbone effect on the persistency could arise from an effectively higher grafting density per unit length in PTD as a result of the compact monomer structure; $b_{0}$ of TD-Gn macromonomer $\left(b_{0}=0.37 \mathrm{~nm}\right)$ is smaller than that of NB-Gn macromonomer $\left(b_{0}=0.5 \mathrm{~nm}\right)$ (see Figure 3 or 2 ). Hence, the grafting density of PTD-Gn is about $25 \%$ larger than that for PNB-Gn. According to Borisov and co-workers, ${ }^{14}$ the ratio $l_{\mathrm{p}} / R_{\mathrm{cs}}$ is an important structural quantity also for nondilute denpols solutions, since it can control the anisotropic excluded volume interactions between denpols, ${ }^{61}$ thereby forming liquid crystalline phase. ${ }^{63}$ Since $l_{\mathrm{p}} / R_{\mathrm{cs}}$ was found to increase with generation for both PTD and PNB backbones, as displayed in Figure 5c, the persistence length should increase faster than $R_{c s}$. However, their values remain below 10, which renders the formation of a liquid crystalline phase

\section{IIV. CONCLUSIONS}

The dendronized PNB containing up to sixth-generation and PTD containing up to fifth-generation dendrons were synthesized by ROMP via grafting-through method for the first time. Successful preparation of unique series of denpols with huge molecular weight up to $1960 \mathrm{kDa}$ enabled us to unravel their structure in solution. The results from SECMALLS, DLS, and SANS experiments can be well understood using a semiflexible cylinder model with three characteristic sizes: contour length, persistence length $l_{\mathrm{p}}$, and cross-sectional radius $R_{\mathrm{cs}}$.

The high-generation denpols display a rod-like conformation, with grafting-induced persistence $l_{\mathrm{p}} \simeq 27 \mathrm{~nm}$ backbone.
The increase of $l_{\mathrm{p}}$ with generation appears to be governed by the molecular weight of the dendron. Compared to the persistence length, the cross-sectional radius $R_{\mathrm{sc}}$ displays a weaker increase with generation, resulting in an increase of aspect ratio $l_{\mathrm{p}} / R_{\mathrm{cs}}$ with generation. Interestingly, the PTD denpols are clearly more persistent than the PNB denpols of the same generation due to the more compact structure and higher grafting density in the PTD. Concerning the variation of the translational diffusion with generation, the shorter G6 $\left(N_{\mathrm{w}}\right.$ $=78)$ is probably closer to a spherical/ovalic rigid object than semirigid chains in conformity with AFM images. For the other samples with sufficiently high contour length, the value of $R_{\mathrm{g}} /$ $R_{\mathrm{h}}$ (Figure 4) conforms to the wormlike chain models.

High-generation denpols are expected to exhibit excluded volume interactions with strength falling between flexible chains and cylindrical nano-objects. The structure and dynamics of such nano-objects in dense states (semidilute solutions to melt) are anticipated to be distinct ${ }^{64-66}$ as estimated from their spatial overlapping and possible formation of liquid crystalline phases.

\section{ASSOCIATED CONTENT}

\section{S Supporting Information}

The Supporting Information is available free of charge on the ACS Publications website at DOI: 10.1021/acs.macromol.9b00457.

Synthetic procedures; NMR spectra; SEC traces; MALDI-TOF spectra; characterization with scattering techniques; and AFM images (PDF)

\section{AUTHOR INFORMATION}

\section{Corresponding Authors}

*E-mail: fabien.dutertre@univ-st-etienne.fr (F.D.).

*E-mail: fytas@mpip-mainz.mpg.de (G. F.).

*E-mail: tlc@snu.ac.kr (T.-L.C.).

ORCID $\odot$

Fabien Dutertre: 0000-0001-5818-7902

Benoit Loppinet: 0000-0003-1855-7619

Sanghee Yang: 0000-0001-7944-6635

Tae-Lim Choi: 0000-0001-9521-6450

\section{Present Address}

${ }^{\nabla}$ Univ Lyon, UJM-Saint-Etienne, CNRS, IMP UMR 5223, F42023 Saint Etienne, France (F.D.).

\section{Author Contributions}

${ }^{\#}$ F.D. and K.-T.B. contributed equally to this work.

\section{Notes}

The authors declare no competing financial interest.

\section{ACKNOWLEDGMENTS}

The authors acknowledge L.L.B - CEA for providing access to the grand instrument, and thank Jacques Jestin for his support regarding SANS experiments and Antje Larsen for technical support in light scattering. GF acknowledges support by ERC AdG SmartPhon (No. 694977). The authors acknowledge the financial support from NRF, Korea, through the following grants: Creative Research Initiative Grant and Nano-Material Technology Program.

\section{REFERENCES}

(1) Macfarlane, R. J.; Kim, B.; Lee, B.; Weitekamp, R. A.; Bates, C. M.; Lee, S. F.; Chang, A. B.; Delaney, K. T.; Fredrickson, G. H.; 
Atwater, H. A.; Grubbs, R. H. Improving Brush Polymer Infrared One-Dimensional Photonic Crystals via Linear Polymer Additives. J. Am. Chem. Soc. 2014, 136, 17374-17377.

(2) Sun, G.; Cho, S.; Clark, C.; Verkhoturov, S. V.; Eller, M. J.; Li, A.; Pavía-Jiménez, A.; Schweikert, E. A.; Thackeray, J. W.; Trefonas, P.; Wooley, K. L. Nanoscopic Cylindrical Dual Concentric and Lengthwise Block Brush Terpolymers as Covalent Preassembled High-Resolution and High-Sensitivity Negative-Tone Photoresist Materials. J. Am. Chem. Soc. 2013, 135, 4203-4206.

(3) Vatankhah-Varnosfaderani, M.; Keith, A. N.; Cong, Y.; Liang, H.; Rosenthal, M.; Sztucki, M.; Clair, C.; Magonov, S.; Ivanov, D. A.; Dobrynin, A. V.; Sheiko, S. S. Chameleon-like elastomers with molecularly encoded strain-adaptive stiffening and coloration. Science 2018, 359, 1509-1513.

(4) Zheng, Y.; Li, S.; Weng, Z.; Gao, C. Hyperbranched polymers: advances from synthesis to applications. Chem. Soc. Rev. 2015, 44, 4091-4130.

(5) Lecommandoux, S.; Chécot, F.; Borsali, R.; Schappacher, M.; Deffieux, A.; Brûlet, A.; Cotton, J. P. Effect of Dense Grafting on the Backbone Conformation of Bottlebrush Polymers: Determination of the Persistence Length in Solution. Macromolecules 2002, 35, 88788881.

(6) Wunderlich, K.; Müllen, K.; Fytas, G. Shape Persistence in Polymers and Supramolecular Assemblies. Conjugated Polymers and Oligomers; World Scientific Publishing, 2018; pp 1-28.

(7) Parshad, B.; Kumari, M.; Achazi, K.; Böttcher, C.; Haag, R.; Sharma, S. K. Chemo-Enzymatic Synthesis of PerfluoroalkylFunctionalized Dendronized Polymers as Cyto-Compatible Nanocarriers for Drug Delivery Applications. Polymers 2016, 8, 311.

(8) Küchler, A.; Adamcik, J.; Mezzenga, R.; Schlüter, A. D.; Walde, P. Enzyme immobilization on silicate glass through simple adsorption of dendronized polymer-enzyme conjugates for localized enzymatic cascade reactions. RSC Adv. 2015, 5, 44530-44544.

(9) Piunova, V. A.; Miyake, G. M.; Daeffler, C. S.; Weitekamp, R. A.; Grubbs, R. H. Highly Ordered Dielectric Mirrors via the SelfAssembly of Dendronized Block Copolymers. J. Am. Chem. Soc. 2013, $135,15609-15616$.

(10) Feng, S.; Xiong, X.; Zhang, G.; Xia, N.; Chen, Y.; Wang, W. Hierarchical Structure in Oriented Fibers of a Dendronized Polymer. Macromolecules 2009, 42, 281-287.

(11) Das, J.; Yoshida, M.; Fresco, Z. M.; Choi, T.-L.; Fréchet, J. M. J.; Chakraborty, A. K. A Dendronized Polymer Is a Single-Molecule Glass. J. Phys. Chem. B 2005, 109, 6535-6543.

(12) Förster, S.; Neubert, I.; Schlüter, A. D.; Lindner, P. How Dendrons Stiffen Polymer Chains: A SANS Study. Macromolecules 1999, 32, 4043-4049.

(13) Zhang, B.; Wepf, R.; Fischer, K.; Schmidt, M.; Besse, S.; Lindner, P.; King, B. T.; Sigel, R.; Schurtenberger, P.; Talmon, Y.; Ding, Y.; Kröger, M.; Halperin, A.; Schlüter, A. D. The Largest Synthetic Structure with Molecular Precision: Towards a Molecular Object. Angew. Chem., Int. Ed. 2011, 50, 737-740.

(14) Mikhailov, I. V.; Darinskii, A. A.; Zhulina, E. B.; Borisov, O. V.; Leermakers, F. A. M. Persistence length of dendronized polymers: the self-consistent field theory. Soft Matter 2015, 11, 9367-9378.

(15) Chang, A. B.; Lin, T.-P.; Thompson, N. B.; Luo, S.-X.; Liberman-Martin, A. L.; Chen, H.-Y.; Lee, B.; Grubbs, R. H. Design, Synthesis, and Self-Assembly of Polymers with Tailored Graft Distributions. J. Am. Chem. Soc. 2017, 139, 17683-17693.

(16) Guo, Y.; van Beek, J. D.; Zhang, B.; Colussi, M.; Walde, P.; Zhang, A.; Kröger, M.; Halperin, A.; Dieter Schlüter, A. Tuning Polymer Thickness: Synthesis and Scaling Theory of Homologous Series of Dendronized Polymers. J. Am. Chem. Soc. 2009, 131, 1184111854.

(17) Grebikova, L.; Kozhuharov, S.; Maroni, P.; Mikhaylov, A.; Dietler, G.; Schluter, A. D.; Ullner, M.; Borkovec, M. The persistence length of adsorbed dendronized polymers. Nanoscale 2016, 8, 1349813506.
(18) Ouali, N.; Méry, S.; Skoulios, A.; Noirez, L. Backbone Stretching of Wormlike Carbosilane Dendrimers. Macromolecules 2000, 33, 6185-6193.

(19) Dutertre, F.; Bang, K.-T.; Loppinet, B.; Choi, I.; Choi, T.-L.; Fytas, G. Structure and Dynamics of Dendronized Polymer Solutions: Gaussian Coil or Macromolecular Rod? Macromolecules 2016, 49, $2731-2740$

(20) Karakaya, B.; Claussen, W.; Gessler, K.; Saenger, W.; Schlüter, A. D. Toward Dendrimers with Cylindrical Shape in Solution. J. Am. Chem. Soc. 1997, 119, 3296-3301.

(21) Helms, B.; Mynar, J. L.; Hawker, C. J.; Fréchet, J. M. J. Dendronized Linear Polymers via "Click Chemistry". J. Am. Chem. Soc. 2004, 126, 15020-15021.

(22) Mynar, J. L.; Choi, T.-L.; Yoshida, M.; Kim, V.; Hawker, C. J.; Frechet, J. M. J. Doubly-dendronized linear polymers. Chem. Commun. 2005, 5169-5171.

(23) Gao, M.; Jia, X.; Kuang, G.; Li, Y.; Liang, D.; Wei, Y. Thermoand $\mathrm{pH}$-Responsive Dendronized Copolymers of Styrene and Maleic Anhydride Pendant with Poly(amidoamine) Dendrons as Side Groups. Macromolecules 2009, 42, 4273-4281.

(24) Gössl, I.; Shu, L.; Schlüter, A. D.; Rabe, J. P. Molecular Structure of Single DNA Complexes with Positively Charged Dendronized Polymers. J. Am. Chem. Soc. 2002, 124, 6860-6865.

(25) Shu, L.; Göossl, I.; Rabe, J. P.; Dieter Schlüter, A. Quantitative Aspects of the Dendronization of Dendronized Linear Polystyrenes. Macromol. Chem. Phys. 2002, 203, 2540-2550.

(26) Shu, L.; Schlüter, A. D.; Ecker, C.; Severin, N.; Rabe, J. P. Extremely Long Dendronized Polymers: Synthesis, Quantification of Structure Perfection, Individualization, and SFM Manipulation. Angew. Chem., Int. Ed. 2001, 40, 4666-4669.

(27) Lee, C. C.; Fréchet, J. M. J. Synthesis and Conformations of Dendronized Poly(1-lysine). Macromolecules 2006, 39, 476-481.

(28) Yoshida, M.; Fresco, Z. M.; Ohnishi, S.; Fréchet, J. M. J. Efficient Divergent Synthesis of Dendronized Polymers with Extremely High Molecular Weight: Structural Characterization by SEC-MALLS and SFM and Novel Organic Gelation Behavior. Macromolecules 2005, 38, 334-344.

(29) Grayson, S. M.; Fréchet, J. M. J. Divergent Synthesis of Dendronized Poly(p-hydroxystyrene). Macromolecules 2001, 34, 6542-6544.

(30) Yu, H.; Schlüter, A. D.; Zhang, B. Synthesis of High Generation Dendronized Polymers and Quantification of Their Structure Perfection. Macromolecules 2014, 47, 4127-4135.

(31) Messmer, D.; Kröger, M.; Schlüter, A. D. Pushing Synthesis toward the Maximum Generation Range of Dendritic Macromolecules. Macromolecules 2018, 51, 5420-5429.

(32) Kasëmi, E.; Zhuang, W.; Rabe, J. P.; Fischer, K.; Schmidt, M.; Colussi, M.; Keul, H.; Yi, D.; Cölfen, H.; Schlüter, A. D. Synthesis of an Anionically Chargeable, High-Molar-Mass, Second-Generation Dendronized Polymer and the Observation of Branching by Scanning Force Microscopy. J. Am. Chem. Soc. 2006, 128, 5091-5099.

(33) Malkoch, M.; Carlmark, A.; Woldegiorgis, A.; Hult, A.; Malmström, E. E. Dendronized Aliphatic Polymers by a Combination of ATRP and Divergent Growth. Macromolecules 2004, 37, 322-329.

(34) Zhang, A.; Zhang, B.; Wächtersbach, E.; Schmidt, M.; Schlüter, A. D. Efficient Synthesis of High Molar Mass, First- to FourthGeneration Distributed Dendronized Polymers by the Macromonomer Approach. Chem. - Eur. J. 2003, 9, 6083-6092.

(35) Kim, K. O.; Choi, T.-L. Synthesis of Rod-Like Dendronized Polymers Containing G4 and G5 Ester Dendrons via Macromonomer Approach by Living ROMP. ACS Macro Lett. 2012, 1, 445-448.

(36) Kang, E.-H.; Lee, I.-H.; Choi, T.-L. Brush Polymers Containing Semiconducting Polyene Backbones: Graft-Through Synthesis via Cyclopolymerization and Conformational Analysis on the Coil-toRod Transition. ACS Macro Lett. 2012, 1, 1098-1102.

(37) Ossenbach, A.; Rüegger, H.; Zhang, A.; Fischer, K.; Schlüter, A. D.; Schmidt, M. Ion-Induced Stretching of Low Generation Dendronized Polymers with Crown Ether Branching Units. Macromolecules 2009, 42, 8781-8793. 
(38) Zhang, A.; Okrasa, L.; Pakula, T.; Schlüter, A. D. Homologous Series of Dendronized Polymethacrylates with a Methyleneoxycarbonyl Spacer between the Backbone and Dendritic Side Chain: Synthesis, Characterization, and Some Bulk Properties. J. Am. Chem. Soc. 2004, 126, 6658-6666.

(39) Cheng, C.; Schmidt, M.; Zhang, A.; Schlüter, A. D. Synthesis of Thermally Switchable Poly(N-isopropylacrylamide-block-dendronized methacrylate)s. Macromolecules 2007, 40, 220-227.

(40) Sun, X.; Lindner, J.-P.; Bruchmann, B.; Schlüter, A. D. Synthesis of Neutral, Water-Soluble Oligo-Ethylene Glycol-Containing Dendronized Homo- and Copolymers of Generations 1, 1.5, 2, and 3. Macromolecules 2014, 47, 7337-7346.

(41) Kim, K. O.; Shin, S.; Kim, J.; Choi, T.-L. Living Polymerization of Monomers Containing endo-Tricyclo[4.2.2.02,5]deca-3,9-diene Using Second Generation Grubbs and Hoveyda-Grubbs Catalysts: Approach to Synthesis of Well-Defined Star Polymers. Macromolecules 2014, 47, 1351-1359.

(42) Kim, K. O.; Choi, T.-L. Synthesis of Dendronized Polymers via Macromonomer Approach by Living ROMP and Their Characterization: From Rod-Like Homopolymers to Block and Gradient Copolymers. Macromolecules 2013, 46, 5905-5914.

(43) Novak, B. M.; Risse, W.; Grubbs, R. H. The Development of Well-Defined Catalysts for Ring-Opening Olefin Metathesis Polymerizations (ROMP). In Polymer Synthesis Oxidation Processes; Springer: Berlin, 1992; Vol. 102, pp 47-72.

(44) Ivin, K. J. M., Mol, J. C. Olefin Metathesis and Metathesis Polymerization; Academic Press: San Diego, 1997.

(45) Buchmeiser, M. R. Homogeneous Metathesis Polymerization by Well-Defined Group VI and Group VIII Transition-Metal Alkylidenes: Fundamentals and Applications in the Preparation of Advanced Materials. Chem. Rev. 2000, 100, 1565-1604.

(46) Yoon, K.-Y.; Suyong, S.; Yong-Jae, K.; Inhye, K.; Eunji, L.; TaeLim, C. One-Pot Preparation of 3D Nano- and Microaggregates via In Situ Nanoparticlization of Polyacetylene Diblock Copolymers Produced by ROMP. Macromol. Rapid Commun. 2015, 36, 10691074.

(47) Slugovc, C. The Ring Opening Metathesis Polymerisation Toolbox. Macromol. Rapid Commun. 2004, 25, 1283-1297.

(48) Anderson, D. R.; Vincent, L.; O’Leary, D. J.; Guy, B.; Grubbs, R. H. Synthesis and Reactivity of Olefin Metathesis Catalysts Bearing Cyclic (Alkyl)(Amino)Carbenes. Angew. Chem., Int. Ed. 2007, 46, $7262-7265$.

(49) Gawin, R.; Tracz, A.; Chwalba, M.; Kozakiewicz, A.; Trzaskowski, B.; Skowerski, K. Cyclic Alkyl Amino Ruthenium Complexes-Efficient Catalysts for Macrocyclization and Acrylonitrile Cross Metathesis. ACS Catal. 2017, 7, 5443-5449.

(50) Kholodenko, A. L. Analytical calculation of the scattering function for polymers of arbitrary flexibility using the Dirac propagator. Macromolecules 1993, 26, 4179-4183.

(51) Kholodenko, A. L. Persistence length and related conformational properties of semiflexible polymers from Dirac propagator. J. Chem. Phys. 1992, 96, 700-713.

(52) Cho, H.; Chung, I.-J. Viscoelastic properties of an isolated polymer chain with arbitrary flexibility, chain length and hydrodynamic interactions from 4-dimensional Dirac propagator. Macromol. Theory Simul. 1999, 8, 279-283.

(53) Lederer, A.; Walther, B.; Anna, K.; Peter, L.; Ralf, S. Is the Universal Law Valid for Branched Polymers? Angew. Chem., Int. Ed. 2013, 52, 4659-4663.

(54) Lederer, A.; Walther, B.; Tobias, H.; Spinelli, H. J.; Nikolay, H.; Andreas, J.; Peter, F.; Ralf, S.; Peter, L. Dendronized Hyperbranched Macromolecules: Soft Matter with a Novel Type of Segmental Distribution. Angew. Chem., Int. Ed. 2015, 54, 12578-12583.

(55) Harnau, L.; Winkler, R. G.; Reineker, P. Dynamic structure factor of semiflexible macromolecules in dilute solution. J. Chem. Phys. 1996, 104, 6355-6368.

(56) Wintermantel, M.; Gerle, M.; Fischer, K.; Schmidt, M.; Wataoka, I.; Urakawa, H.; Kajiwara, K.; Tsukahara, Y. Molecular Bottlebrushes. Macromolecules 1996, 29, 978-983.
(57) Harnau, L.; Winkler, R. G.; Reineker, P. Dynamic properties of molecular chains with variable stiffness. J. Chem. Phys. 1995, 102, $7750-7757$.

(58) Zhulina, E. B.; Sheiko, S. S.; Borisov, O. V. Solution and Melts of Barbwire Bottlebrushes: Hierarchical Structure and Scale-Dependent Elasticity. Macromolecules 2019, 52, 1671.

(59) Hsu, H.-P.; Paul, W.; Rathgeber, S.; Binder, K. Characteristic Length Scales and Radial Monomer Density Profiles of Molecular Bottle-Brushes: Simulation and Experiment. Macromolecules 2010, 43, $1592-1601$.

(60) Paturej, J.; Sheiko, S. S.; Panyukov, S.; Rubinstein, M. Molecular structure of bottlebrush polymers in melts. Sci..Adv. 2016, 2, No. e1601478.

(61) Feuz, L.; Leermakers, F. A. M.; Textor, M.; Borisov, O. Bending Rigidity and Induced Persistence Length of Molecular Bottle Brushes: A Self-Consistent-Field Theory. Macromolecules 2005, 38, 88918901.

(62) Kroeger, A.; Zhang, B.; Rosenauer, C.; Schlüter, A. D.; Wegner, G. Solvent induced phenomena in a dendronized linear polymer. Colloid Polym. Sci. 2013, 291, 2879-2892.

(63) Egorov, S. A.; Milchev, A.; Binder, K. Capillary Nematization of Semiflexible Polymers. Macromol. Theory Simul. 2017, 26, No. 1600036.

(64) Costanzo, S.; Scherz, L. F.; Schweizer, T.; Kröger, M.; Floudas, G.; Schlüter, A. D.; Vlassopoulos, D. Rheology and Packing of Dendronized Polymers. Macromolecules 2016, 49, 7054.

(65) Scherz, L. F.; Costanzo, S.; Huang, Q.; Schlüter, A. D.; Vlassopoulos, D. Dendronized Polymers with Ureidopyrimidinone Groups: An Efficient Strategy To Tailor Intermolecular Interactions, Rheology, and Fracture. Macromolecules 2017, 50, 5176-5187.

(66) Qian, Z.; Koh, Y. P.; Pallaka, M. R.; Chang, A. B.; Lin, T.-P.; Guzmán, P. E.; Grubbs, R. H.; Simon, S. L.; McKenna, G. B. Linear Rheology of a Series of Second-Generation Dendronized Wedge Polymers. Macromolecules 2019, 52, 2063-2074. 